Pacific Journal of Mathematics

PSEUDOCOMPACT GROUP TOPOLOGIES AND TOTALLY 


\title{
PSEUDOCOMPACT GROUP TOPOLOGIES AND TOTALLY DENSE SUBGROUPS
}

\author{
W. W. COMFort and T. Soundararajan
}

Throughout this synopsis all topologies are Hausdorff topological group topologies, and $\langle G, \mathscr{T}\rangle$ is assumed compact. The symbol $w$ denotes weight. Definition. A subgroup $H$ of $\langle G, \mathscr{T}\rangle$ is totally dense (in $G$ ) if $H \cap K$ is dense in $K$ for every closed subgroup $K$ of $G$. We prove these results.

If $\mathscr{T}^{\prime} \supsetneqq \mathscr{T}$ with $\left\langle G, \mathscr{T}^{\prime}\right\rangle$ pseudocompact, then not every $\mathscr{T}^{\prime}$-closed subgroup of $G$ is $\mathscr{T}$-closed. If $w(G, \mathscr{T})>\omega$ with $\langle G, \mathscr{T}\rangle$ totally disconnected Abelian, then there is pseudocompact $\mathscr{T}^{\prime} \supsetneqq \mathscr{T}$.

Not every infinite $\langle G, \mathscr{T}\rangle$ has a proper, totally dense subgroup. But (a) if $w\langle G, \mathscr{T}\rangle\rangle \omega$ with $\langle G, \mathscr{T}\rangle$ connected Abelian, or (b) if $\langle G, \mathscr{T}\rangle$ is totally disconnected Abelian and in the dual group $p$-primary decomposition $\hat{G}=\oplus_{p} \hat{G}_{p}$ one has $\left|\hat{G}_{p}\right|>\omega$ for infinitely many primes $p$, then $\langle G, \mathscr{I}\rangle$ has a proper, totally dense, pseudocompact subgroup.

Let $H$ be a totally dense subgroup of $\langle G, \mathscr{T}\rangle$. Then (a) $|G| \leqq 2^{|H|}$; (b) if $G$ is Abelian then $|G| \leqq|H|^{\omega}$; (c) if $G$ is connected Abelian then $|G|=|H|$; (d) if $G$ is totally disconnected and $H$ countably compact, then $G=H$; (e) there are examples with $\langle G, \mathscr{T}\rangle$ (totally disconnected) Abelian and $|H|<|G|$.

1. Introduction and motivation. All the topological groups hypothesized in this paper are assumed to satisfy the $T_{0}$ separation property; as is well-known (see for example [18] (Theorem 8.4)), this guarantees that they are in fact completely regular, Hausdorff spaces, i.e., Tychonoff spaces.

The authors' interests in pseudocompact group topologies (defined below) arise, quite independently and to our surprise, from distinct and differing considerations not related at first glance: (a) as a natural class of spaces, considerably broader than the class of compact spaces, with fascinating topological features; and (b) as important tools in the development of topological Galois theory. As to (a) we note that major portions of [33], [20], [34] are devoted to questions concerning the existence and the size of dense pseudocompact subgroups of compact groups, while [7] shows that the product of pseudocompact groups is pseudocompact and that the

1 The first author gratefully acknowledges support received from the National Science Foundation under grant NSF-MCS78-00912.

2 The second author is grateful to the INDO-US Subcommission on Education and Culture, U.S.A. and to the University Grants Commission of India for a Visitorship to U.S.A. which enabled this collaboration. 
pseudocompact groups are exactly those whose Stone-Čech compactification is a compact group extension. As to (b) we cite only [26], [28], and from [29] the following result: for a normal, separable, algebraic extension $E$ of a field $K$, the dense, pseudocompact subgroups of the (compact) Galois group $G=G(E / K)$ are exactly those subgroups $H$ with the property that every $K$-isomorphism: $F \rightarrow E$ with $F$ countably generated over $K$ extends to an element of $H$.

The question of altering the topology of a topological group without altering the family of closed subgroups also comes to our attention from two different directions - as a question of interest in its own right in the context of harmonic analysis and, again, as a question with applications in topological Galois theory. In the latter connection, see [28] and [21]. In the former we recall that K. A. Ross [25] has shown that if $\mathscr{T}$ and $\mathscr{T}^{\prime}$ are locally compact group topologies for Abelian $G$ with $\mathscr{T}$ compact such that $\langle G, \mathscr{T}\rangle$ and $\left\langle G, \mathscr{T}^{\prime}\right\rangle$ have the same closed subgroups, then $\mathscr{T}=\mathscr{T}^{\prime}$ if either $\mathscr{T}$ is totally disconnected or $\mathscr{T}^{\prime} \supset \mathscr{T}$; several of the results of [25] have been extended to the general (non-Abelian) setting by N. W. Rickert [24] and by M. Rajagopalan [23].

The authors are indebted to James D. Reid for several valuable conversations and suggestions, and to the referee for a helpful report.

2. Notation, definitions and results from the literature. Here for convenient reference we collect a number of results to be used without proof later in this paper. In general, our notations and conventions are guided by those of Hewitt and Ross [18], which is our principal reference.

We denote by $\omega$ the least infinite cardinal. For an infinite cardinal $\alpha$, the symbol $\alpha^{+}$denotes the least cardinal larger than $\alpha$.

The symbols $\mathbf{Z}, \mathbf{R}$ and $\mathbf{T}$ denote the integers, the real numbers, and the circle, respectively; in each case the usual algebraic and topological properties are assumed when necessary.

The symbol $\simeq$ denotes group isomorphism; the statement $G \simeq \widetilde{G}$ has no topological import. The symbol $\cong$ denotes topological group isomorphism; that is, we write $G \cong \widetilde{G}$ only if $G$ and $\widetilde{G}$ are topological groups and there is a one-to-one function from $G$ onto $\widetilde{G}$ which is simultaneously a group isomorphism and a topological homeomorphism. We occasionally use the symbol $=$ in contexts where rigor, perhaps, demands $\cong$.

We often use the symbol $e$ to denote the identity element of a group.

For an Abelian topological group $G$ we denote by $\hat{G}$ the set of continuous homomorphisms from $G$ to the circle group T. When $G$ 
is locally compact, as in (2.1) and (2.2) below, $\hat{G}$ carries the compactopen topology; otherwise, as in (2.8), $\hat{G}$ denotes simply the (nontopologized) group. For $\left\{G_{i}: i \in I\right\}$ a set of groups we set

$$
\bigoplus_{i \in I} G_{i}=\left\{x \in \prod_{i \in I} G_{i}:\left|\left\{i \in I: x_{i} \neq e_{i}\right\}\right|<\omega\right\} .
$$

(2.1) Let $\left\{G_{i}: i \in I\right\}$ be a set of compact Abelian groups, $G=$ $\prod_{i \in I} G_{i}$ and $H=\bigoplus_{i \in I} G_{i}$ with $H$ discrete. Then

(a) $\hat{G} \cong \bigoplus_{i \in I} \hat{G}_{i}$ with $\hat{G}$ discrete, and

(b) $\hat{H} \cong \prod_{i \in I} \hat{G}_{i}$.

(2.2) [See [18], 23.25 and 24.11.] Let $G$ be a locally compact Abelian group, $H$ a closed subgroup and $A$ the annihilator in $\hat{G}$ of $H$ (that is,

$$
A=\{\chi \in \hat{G}: \chi(x)=1 \text { for all } x \in H\}) .
$$

Then $(G / H)^{\wedge} \cong A$ and $\hat{H} \cong \hat{G} / A$.

(2.3) [See [18], 24.47.] Let $G$ be an Abelian topological group.

(a) If $G$ is discrete, then $|\widehat{G}|=2^{|G|}$;

(b) If $G$ is compact, then $|G|=2^{|\hat{G}|}$.

For a space $X$ and $x \in X$, we denote by $w X$ and $w(X, x)$ the weight of $X$ and the local weight of $X$ at $x$, respectively. It is easy to prove that if $X$ is locally compact (at $x$ ) then $w(X, x)$ is equal to the pseudoweight $\psi(X, x)$ of $X$ at $x$; this is (by definition) the least cardinal number which is the cardinal of a family of open subsets of $X$ whose intersection is equal to $\{x\}$. The following result may be viewed as a generalization of (2.3).

(2.4) [See [19], 28.58(b) for $G$ compactly generated; or [2], 2.1.] Let $G$ be a nondiscrete, locally compact, $\sigma$-compact group. Then $w G=w(G, e)$ and $|G|=2^{w G}$.

(2.5) [See [18], 24.25.] Let $G$ be a compact, Abelian group. Then $G$ is connected if and only if $\hat{G}$ is torsion-free.

(2.6) [See [18], 8.7.] Let $G$ be a compact group and $\left\{U_{n}: n<\omega\right\}$ a countable set of neighborhoods of $e$. There is a compact, normal, $G_{o}$ subgroup $N$ of $G$ such that $N \subset \bigcap_{n<\omega} U_{n}$.

For a topological space $X$ we denote by $C^{*}(X)$ the set of bounded, real-valued continuous functions on $X$. The space $X$ is said to be pseudocompact if every continuous, real-valued function on $X$ is in $C^{*}(X)$; for $X$ a Tychonoff space, this is equivalent to the condition that every locally finite family of nonempty, open subsets of $X$ is finite.

For a Tychonoff space $X$ we denote by $\beta X$ the Stone-Čech compactification of $X$.

(2.7) [See [17], 28.] A Tychonoff space $X$ is pseudocompact if and only if $X$ meets each nonempty $G_{\delta}$ subset of $\beta X$. 
A topological group $G$ is said to be totally bounded if for every nonempty open $U \subset G$ there is finite $F \subset G$ such that $G=F U$. It is clear that every compact group, and each of its subgroups, is totally bounded. Conversely Weil [32] has shown that every totally bounded group $G$ is dense in an essentially unique compact group $\bar{G}$ (called the Weil completion of $G$ ). Throughout this paper we use the symbol $\bar{G}$ only when $G$ is a totally bounded group and $\bar{G}$ is its Weil completion.

The symbol $G_{d}$ denotes the group $G$ topologized with the discrete topology.

(2.8) [See [6].] If $G$ is an Abelian group, $\mathscr{H}$ a point-separating subgroup of $\hat{G}_{d}$ and $\mathscr{T}(\mathscr{H})$ the topology induced on $G$ by $\mathscr{H}$, then $\langle G, \mathscr{T}(\mathscr{H})\rangle$ is a totally bounded group and $\langle G, \mathscr{T}(\mathscr{H})\rangle^{\wedge}=\mathscr{H}$. Conversely if $\langle G, \mathscr{T}\rangle$ is a tatally bounded Abelian group and $\mathscr{H}=$ $\langle G, \mathscr{T}\rangle^{\wedge}$, then $\mathscr{T}=\mathscr{T}(\mathscr{H})$.

(2.9) [See [7], 1.1.] Every pseudocompact group is totally bounded.

(2.10) [See [7], 1.2.] For a totally bounded group $G$, the following are equivalent:

(a) $G$ is pseudocompact;

(b) $\bar{G}=\beta G$;

(c) every translate in $\bar{G}$ of every closed, normal $G_{\delta}$ subgroup of $\bar{G}$ meets $G$.

For $\left\{X_{i}: i \in I\right\}$ a set of spaces, $p_{i} \in X_{i}$ and $p=\left\langle p_{i}: i \in I\right\rangle \in \prod_{i \in I} X_{i}$, the $\Sigma$-product of the family $\left\{X_{i}: i \in I\right\}$ at $p$ is the family

$$
\Sigma=\left\{x \in \prod_{i \in I} X_{i}:\left|\left\{i \in I: x_{i} \neq p_{i}\right\}\right| \leqq \omega\right\} .
$$

It is clear that if each $X_{i}$ is a (topological) group and $p_{i}=e_{i}$, then the $\Sigma$-product is also a topological group.

(2.11) [See [9], [14] or [22].] Let $\left\{G_{i}: i \in I\right\}$ be a set of compact groups and $\Sigma$ the $\Sigma$-product of $\left\{G_{i}: i \in I\right\}$ at $e$. Then $\Sigma$ is a countably compact (hence, pseudocompact) topological group.

We set $\mathbf{P}=\{p \in Z: p$ is prime $\}$. For $p \in \mathbf{P}$ we denote by $Z(p)$ the cyclic group of order $p$. A group $G$ is a $p$-group (or, a $p$ primary group) if the order of each of its elements is a power of $p$.

(2.12) [See [18], A.3.] Let $G$ be an Abelian torsion group and for $p \in \mathbf{P}$ let $G_{p}$ be the set of elements of $G$ whose order is a power of $p$. Then $G_{p}$ is a $p$-primary subgroup of $G$, and $G$ is isomorphic to the group $\bigoplus_{p \in \mathrm{P}} G_{p}$.

(2.13) [See [18], A.11(III).] Let $G$ be an Abelian $p$-group with socle $S=\{x \in G$ : order of $x=p\} \cup\{e\}$, for $x \in G$ let $p^{k(x)}$ be the order of $x$ in $G$ and let $L$ be a maximal independent set in $G$. Then $\left\{p^{k(x)-1} x: x \in L\right\}$ is a basis for $S$. 
(2.14) Corollary. Let $G$ be an Abelian p-group with $|G|>\omega$ and let $S$ be the socle of $G$. Then $|S|=|G|$.

Proof. With $L$ as in (2.13) we have $|G| \leqq|L| \cdot \omega$ and $|S| \geqq|L|$; from $|G|>\omega$ follows $|S|=|G|$, as required.

For an Abelian group $G$, we denote by $t G$ or $t(G)$ the torsion subgroup of $G$.

3. Larger topologies with no new closed subgroups. We show in this section (Theorem 3.5) that if a preudocompact group topology $\mathscr{T}^{\prime}$ is strictly finer than some compact group topology $\mathscr{T}$, then not every $\mathscr{T}^{\prime}$-closed subgroup is $\mathscr{T}$-closed.

In the landmark paper [17] in which pseudocompact spaces (inter alia) were first introduced, Hewitt showed (Theorem 30) that a normal, pseudocompact Tychonoff space is countably compact (the terminology of [17] differs from ours today); in particular, a pseudocompact metric space is compact. It is tempting then to conjecture that a first-countable, pseudocompact space is compact, but the Katětov-Isbell space $\psi$ described in Problem 5I of [13] is a counterexample; indeed the space $\psi$ is locally compact and every subset of $\psi$ is a $G_{j}$. We show now that for pseudocompact groups, and for their quotients by (not necessarily normal) closed subgroups, metrizability is equivalent to the condition that every point is a $G_{\delta}$.

LEMMA 3.1. Let $G$ be a pseudocompact group in which each point is $a G_{j}$. Then $G$ is a compact metric space.

Proof. It is enough to show that $\{e\}$ is a $G_{\delta}$ in the Weil completion $\bar{G}$ of $G$; for then $\bar{G}$ is metrizable ([18] (Theorem 8.5)) and hence as noted above $G$, a pseudocompact metric space, is itself compact metric.

Since $\bar{G}=\beta G(2.10)$ there is $f \in C^{*}(\bar{G})$ such that $f^{-1}(\{0\}) \cap G=\{e\}$. If there is $x \in f^{-1}(\{0\}) \backslash G$ we choose $g \in C^{*}(\bar{G})$ with $g \geqq 0, g(x)=0$ and $g(e)>0$, and we set $h=|f|+g$. Then $h>0$ on $G$ and from $h(x)=0$ we have $\inf (h \mid G)=0$; this contradicts the hypothesis that $G$ is pseudocompact.

LEMMA 3.2. Let $G$ be a totally bounded group. If $G / N$ is compact for every closed, normal, $G_{o}$ subgroup $N$ of $G$, then $G$ is pseudocompact.

Proof. It is by (2.10) enough to show that if $H$ is a closed, normal, $G_{\delta}$ subgroup of the Weil completion $\bar{G}$ and if $x \in \bar{G}$, then $x H \cap G \neq \phi$. We set $N=H \cap G$ and we note that $N$ is a closed, 
normal, $G_{j}$ subgroup of $G$ and (with $\varphi$ the natural homomorphism from $\bar{G}$ onto $\bar{G} / H$ ) that

$$
\operatorname{ker}(\varphi \mid G)=\operatorname{ker}(\varphi) \cap G=H \cap G=N .
$$

The group $\varphi[G]$, which is dense in $\varphi[\bar{G}]$, is (isomorphic to) the compact group $G / N$ and hence $\varphi[G]=\bar{G} / H$; that is, for $x \in \bar{G}$ there is $g \in G$ such that $x H=g H$, as required.

Combining the results of Lemmas 3.1 and 3.2 , we obtain a characterization of pseudocompact groups which we have not found elsewhere in the literature. We note that in what follows it is not assumed that the group $G$ is totally bounded, nor is it assumed that the subgroups $H$ of conditions (b) and (c) are normal in $G$; of course additional equivalent conditions can be obtained by imposing this additional hypothesis in (b) or (c).

THEOREM 3.3. Let $G$ be a topological group. The following staiements are equivalent.

(a) $G$ is pseudocompact;

(b) for every closed, $G_{o}$ subgroup $H$ of $G$ the coset space $G / H$ is compact metric;

(c) for every closed, $G_{\tilde{o}}$ subgroup $H$ of $G$ the coset space $G / H$ is compact.

Proof. (a) $\Rightarrow$ (b) The group $G$ is totally bounded (2.9) and there is a sequence $\left\{U_{n}: n<\omega\right\}$ of open subsets of $\bar{G}$ such that $H=$ $\left(\cap_{n<\omega} U_{n}\right) \cap G$. There is by (2.6) a compact, normal, $G_{\delta}$ subgroup $\widetilde{N}$ of $\bar{G}$ such that $\tilde{N} \subset \bigcap_{n<\omega} U_{n}$; we set $N=\tilde{N} \cap G$. Then $\{N\}$ is a $G_{i}$ in the pseudocompact group $G / N$, so $G / N$ is a compact metric space (by Lemma 3.1 above, applied to $G / N$ in place of $G$ ). The natural function from the group $G / N$ onto the space $G / H$ is easily seen to be continuous. Thus $G / H$, like every (Hausdorff) continuous image of a compact metric space, is itself compact metric ([11] (Corollary 2, page 105)).

(b) $\Rightarrow$ (c) This is obvious.

(c) $\Rightarrow$ (a) It is by Lemma 3.2 enough to show that $G$ is totally bounded. Let $U$ be a neighborhood of $e$, set $V_{0}=U$, recursively for $n<\omega$ let $V_{n}$ be a symmetric neighborhood of $e$ such that

$$
c l V_{n} \subset V_{n} V_{n}^{-1} \subset V_{n-1},
$$

and set $H=\bigcap_{n<\omega} V_{n}$. Then $H$ is a closed, $G_{o}$ subgroup of $G$ and the natural function $\varnothing$ from $G$ onto the compact space $G / H$ is open. Hence the (open) cover $\left\{\varphi\left[x V_{1}\right]: x \in G\right\}$ of $G / H$ has a finite subcover $\left\{\varphi\left[x_{i} V_{1}\right]: 1 \leqq i \leqq n\right\}$ and we have 


$$
G=\bigcup_{i=1}^{n} x_{i} V_{1} H \subset \bigcup_{i=1}^{n} x_{i} V_{1} V_{1}^{-1} \subset \bigcup_{i=1}^{n} x_{i} U
$$

as required.

Lemma 3.4. Let $G$ be a totally bounded group and $U$ a neighborhood of $e$. There are a closed, normal $G_{o}$ subgroup $N$ of $G$ and a neighborhood $V$ of e such that $V N \subset U$.

Proof. Let $\widetilde{U}$ and $\widetilde{V}$ be neighborhoods of $e$ in $\bar{G}$ such that $\widetilde{U} \cap G=U$ and $\widetilde{V}^{2} \subset \widetilde{U}$. There is by (2.6) a closed, normal, $G_{\delta}$ subgroup $\tilde{N}$ of $\bar{G}$ such that $\tilde{N} \subset \tilde{V}$. We set $N=\tilde{N} \cap G$ and $V=\widetilde{V} \cap G$; it is clear that $N$ and $V$ are as required.

We give the principal result of this section.

Theorem 3.5. Let $\langle G, \mathscr{T}\rangle$ and $\left\langle G, \mathscr{T}^{\prime}\right\rangle$ be topological groups with the same closed subgroups such that $\langle G, \mathscr{T}\rangle$ is compact, $\langle G$, $\left.\mathscr{T}^{\prime}\right\rangle$ is pseudocompact, and $\mathscr{T}^{\prime} \supset \mathscr{T}$. Then $\mathscr{T}=\mathscr{T}^{\prime}$.

Proof. Let $e \in U \in \mathscr{T}^{\prime}$. Since $\left\langle G, \mathscr{T}^{\prime}\right\rangle$ is totally bounded there are by Lemma 3.4 a $\mathscr{T}^{\prime}$-closed, $\mathscr{T}^{\prime}-G_{\delta}$ normal subgroup $N$ of $G$ and $V \in \mathscr{T}^{\prime}$ such that $V N \subset U$.

Since $\mathscr{T}^{\prime} \supset \mathscr{T}$, the quotient topology $\mathscr{T}_{q}^{\prime}$ induced on $G / N$ by $\mathscr{T}^{\prime}$ contains the quotient topology $\mathscr{T}_{q}$ induced on $G / N$ by $\mathscr{T}$. The former is compact (by Theorem 3.3) and the latter is Hausdorff (since $N$ is $\mathscr{T}$-closed). Thus $\mathscr{T}_{q}^{\prime}=\mathscr{T}_{q}$ and from $V N=\varphi[V] \in \mathscr{T}_{q}^{\prime}$ we have $V N \in \mathscr{T}_{q}$. It follows that

$$
\varphi^{-1}(\varphi[V])=V N \in \mathscr{T} .
$$

Thus every $\mathscr{T}^{\prime}$-neighborhood of $e$ contains a $\mathscr{T}$-neighborhood of $e$ and we have $\mathscr{T}^{\prime} \subset \mathscr{T}$, as required.

We note that (as the proof above shows) it is not necessary to assume that every $\mathscr{T}^{\prime}$-closed subgroup is $\mathscr{T}$-closed, or that every $\mathscr{T}^{\prime}$-closed-G $G_{\delta}$ subgroup is $\mathscr{T}$-closed- $G_{\delta}$; it is enough to assume that every $\mathscr{T}^{\prime}$-closed, $\mathscr{T}^{\prime}-G_{i}$, normal subgroup of $G$ is $\mathscr{T}$-closed.

4. Larger pseudocompact group topologies. We have seen that a compact group topology cannot extend properly to a pseudocompact group topology with the same set of closed subgroups. We show in this section (Theorem 4.4) that under suitable conditions a compact group topology does extend properly to a pseudocompact group topology (in which, of course, certain subgroups not previously closed become closed). Our argument, which evidently is applicable only in special circumstances, is easily summarized: Given 
compact $\langle G, \mathscr{T}\rangle$ we find a proper (dense) pseudocompact subgroup $H$ of $G$ with $|G / H|<\omega$ and we take for $\mathscr{T}^{\prime}$ the topological group topology generated by $\mathscr{T}$ and $H$.

We begin with two lemmas. Part (c) of Lemma 4.1 is a trifle out of place here: it belongs below in $\S 5$. We prove 4.1(c) now because it is immediate from 4.1(a), which is needed immediately.

Definition. A subgroup $H$ of a topological group $G$ is totally dense (in $G$ ) if $c l_{G}(H \cap K)=K$ for every closed subgroup $K$ of $G$.

LEMMA 4.1. Let $G$ and $\widetilde{G}$ be compact groups, $\varphi$ a continuous homomorphism from $G$ onto $\widetilde{G}, \widetilde{H}$ a subgroup of $\widetilde{G}$ and $H=\varphi^{-1}(\widetilde{H})$.

(a) If $D$ is dense in $\widetilde{H}$, then $\Phi^{-1}(D)$ is dense in $H$.

(b) If $\tilde{H}$ is pseudocompact, then $H$ is pseudocompact.

(c) If $\widetilde{H}$ is totally dense in $\widetilde{G}$, then $H$ is totally dense in $G$.

Proof. It follows from the Baire category theorem that $\phi$ is an open function (cf. [18] (Theorem 5.29)). Hence $\varphi \mid H$ is open: If $U$ is open in $H$ and $V$ is an open subset of $G$ such that $V \cap H=U$, then $\varphi[U]=\varphi[V] \cap \widetilde{H}$ and hence $\varphi[U]$ is open in $\widetilde{H}$.

(a) If $U$ is a nonempty, open subset of $H$ then $\varphi[U] \cap D \neq \phi$ and hence $U \cap \varphi^{-1}(D) \neq \phi$, as required.

(b) Suppose there is an (infinite) locally finite family $\left\{U_{n}: n<\omega\right\}$ of nonempty, open subsets of $H$. We obtain the required contradiction by showing that for every $p \in \widetilde{H}$ some neighborhood $U$ of $p$ meets $\varphi\left[U_{n}\right]$ for only finitely many $n<\omega$. We note first that $\varphi^{-1}(\{p\})$ is a compact subset of $G$ and is a subset of $H$. Since $\left\{U_{n}: n<\omega\right\}$ is locally finite in $H$ and $\varphi^{-1}(\{p\})$ is compact there is an open subset $V$ of $H$ such that $V \cap U_{n} \neq \phi$ for only finitely many $n<\omega$. There is a neighborhood $W$ of $e$ in $H$ such that $\varphi^{-1}(\{p\})$. $W \subset V$; we set $U=p \cdot \varphi[W]$ and we see for $N<\omega$ that if there are $w \in W$ and $u_{n} \in U_{n}$ with $p \cdot \varphi(w)=\varphi\left(u_{n}\right)$ then with $q=u_{n} w^{-1}$ we have $q \in \varphi^{-1}(\{p\})$ and hence

$$
V \cap U_{n} \supset\left(\varphi^{-1}(\{p\}) \cdot W\right) \cap U_{n} \neq \phi .
$$

(c) Let $K$ be a closed subgroup of $G$ and set $\psi=\varphi \mid K$. Then $H \cap \psi[K]$ is dense in $\psi[K]$ and it follows from part (a), with $G, \widetilde{G}$ $\varphi, \widetilde{H}$ and $H$ replaced by $K, \psi[K], \psi, \widetilde{H} \cap \psi[K]$ and $H \cap K$, respectively, that the set $\psi^{-1}(\widetilde{H} \cap \psi[K])$, which is $H \cap K$, is dense in $K$.

Lemma 4.2. Let $G=Z(p)^{\alpha}$ with $p \in \boldsymbol{P}$ and $\alpha>\omega$. The group $G$ has a dense, pseudocompact subgroup $H$ with $|G / H|=p$.

Proof. As in (2.11) we set 


$$
\Sigma=\left\{x \in G:\left|\left\{\xi<\alpha: x_{\xi} \neq 0\right\}\right| \leqq \omega\right\} ;
$$

then $\Sigma$ is a dense, pseudocompact (indeed, countably compact) subgroup of $G$. Let $x \in G$ with $x \notin \Sigma$ and let $H$ be a subgroup of $G$ maximal with respect to the property that $H \supset \Sigma$ and $x \notin H$. It is clear that $\Sigma$ (and hence $H$ ) is dense in $G$, and that $|G / H|=p$. Like any space with a dense, pseudocompact subspace, $H$ itself is pseudocompact.

THEOREM 4.3. Let $\langle G, \mathscr{T}\rangle$ be a compact, totally disconnected, Abelian group with $w(G)>\omega$. Then there is a dense, pseudocompact subgroup $H$ of $G$ such that $1<|G / H|<\omega$.

Proof. The dual group $\hat{G}$ of $G$ is a torsion group; we write $\hat{G}=\bigoplus_{p \in P} \hat{G}_{p}$ with $\hat{G}_{p}$ the $p$-primary subgroup of $\hat{G}$. Since $|\hat{G}|=$ $w(G)>\omega$ there is $p \in \mathbf{P}$ such that $\left|\hat{G}_{p}\right|>\omega$. We denote by $S$ the socle of $\hat{G}_{p}$, that is, we write

$$
S=\left\{\chi \in \widehat{G}_{p}: \text { order of } \chi=p\right\} \cup\{e\},
$$

and we note from (2.14) that $|S|>\omega$ and that $S$ is (isomorphic to) the weak sum $S=\bigoplus_{i \in I} Z(p)$ with $|I|=\alpha>\omega$. With $A$ the annihilator of $S$ in $G$ it follows from (2.2) that there is a continuous onto homomorphism

$$
\varphi: G \longrightarrow G / A \cong \hat{S} \cong \prod_{i \in I}(Z(p))^{\wedge} \cong(Z(p))^{\alpha},
$$

and from Lemma 4.2 there is a dense, pseudocompact subgroup $\widetilde{H}$ of $G / A$ with $|(G / A) / \widetilde{H}|=p$. We set $H=\varphi^{-1}(\widetilde{H})$ and we note from Lemma 4.1 that $H$ is a dense, pseudocompact subgroup of $G$; it is clear that $|G / H|=p$.

THEOREM 4.4. Let $\langle G, \mathscr{T}\rangle$ be a compact, totally disconnected Abelian group with $w(G)>\omega$. Then there is a pseudocompact group topology $\mathscr{T}^{\prime}$ for $G$ such that $\mathscr{T}^{\prime} \supseteqq \mathscr{T}$.

Proof. There is by Theorem 4.3 a dense, pseudocompact subgroup $H$ of $G$ with $1<|G| H \mid<\omega$. Let $\mathscr{T}^{\prime}$ be the topology for $G$ generated by $\mathscr{T}$ and the finite family $\{x H: x \in G\}$; that is, for $A \subset G$ we have $A \in \mathscr{T}^{\prime}$ if and only if for each $x \in G$ the set $A \cap x H$ is open in $x H$ in the topology induced on $x H$ by $\mathscr{T}$. It is clear that $\left\langle G, \mathscr{T}^{\prime}\right\rangle$ is a topological group in which $G$, like any finite union of pseudocompact spaces, is pseudocompact.

REMARKs 4.5. (a) If in Theorem 4.4 the hypothesis $w(G)>\omega$ is omitted the resulting statement fails (for every compact group 
$\langle G, \mathscr{T}\rangle$ with $w G \leqq \omega)$. Indeed for $\mathscr{T}^{\prime} \supset \mathscr{T}$ every point of $G$ is a $\mathscr{T}-G_{\hat{o}}$ and hence a $\mathscr{T}^{\prime}-G_{\dot{\delta}}$, and if follows from Lemma 3.1 that if $\left\langle G, \mathscr{T}^{\prime}\right\rangle$ is a pseudocompact group then $\left\langle G, \mathscr{T}^{\prime}\right\rangle$ is compact and hence $\mathscr{T}^{\prime}=\mathscr{T}$.

(b) In an early version of this manuscript (see also the abstract [8]) the authors conjectured that the group $T^{\left(\omega^{+}\right)}$in its usual compact, connected topology admits no proper pseudocompact topological group extension. Subsequently Lewis C. Robertson and the firstlisted author have shown the conjecture false; it follows that Theorem 4.4 remains valid if the hypothesis that $\langle G, \mathscr{T}\rangle$ is totally disconnected is omitted. Details will appear in [4].

We show below in Theorem 7.2 that there are many totally bounded group topologies on the circle whose closed subgroups are exactly the usual closed (compact) subgroups. (In view of Theorem 3.5, no such topology extending the usual topology can be pseudocompact.) We show now, denoting by $\mathscr{B}(\mathscr{T})$ the class of totally bounded topologies $\mathscr{T}^{\prime}$ for an Abelian group $\langle G, \mathscr{G}\rangle$ for which $\mathscr{T}$ and $\mathscr{T}^{\prime}$ have the same closed subgroups, that the partially ordered set $\mathscr{B}\left(\mathscr{S}^{-}\right)$has maximal elements.

THEOREM 4.6. Let $\langle G, \mathscr{T}\rangle$ be a totally bounded Abelian group. For every $\mathscr{G}^{\prime} \in \mathscr{B}(\mathscr{S})$ there is $\mathscr{S} \in \mathscr{B}(\mathscr{G})$ with $\mathscr{T} \subset \mathscr{S}$ such that $\mathscr{S}$ is maximal in the following sense: If $\mathscr{S}^{\prime} \in \mathscr{B}(\mathscr{T})$ and $\mathscr{S} \subset \mathscr{S}^{\prime}$, then $\mathscr{S}=\mathscr{S}^{\prime}$.

Proof. It is enough to show that every chain $\left\{\mathscr{S}_{2}: \lambda \in \Lambda\right\}$ in $\mathscr{B}(\mathscr{S})$ has an upper bound in $\mathscr{B}(\mathscr{S})$. Set $\mathscr{S}=\mathbf{U}_{\lambda \in i} \mathscr{S}_{i}$, let $\mathscr{I}(\lambda)$ be the set of $\mathscr{S}_{\lambda}$-continuous homomorphisms into the circle (i.e., $\left.\mathscr{I}(\lambda)=\left\langle G, \mathscr{S}_{\lambda}\right\rangle^{\wedge}\right)$ and set $\mathscr{S}=\bigcup_{\lambda \in} \mathscr{I}(\lambda)$. We have from (2.8) that $\mathscr{I}=\langle G, \mathscr{S}\rangle \wedge$ and that $\langle G, \mathscr{S}\rangle$ is totally bounded.

It remains to show $\mathscr{S} \in \mathscr{B}(\mathscr{S})$. (The following argument is taken in all essentials from [21] (Lemma 2.2).) Let $H$ be an $\mathscr{S}$ closed subgroup of $G$, let $\bar{G}$ denote the (compact, Abelian) Weil completion of $\langle G, \mathscr{S}\rangle$ and set $\bar{H}=c l_{\bar{G}} H$. Writing

$$
A=\left\{\chi \in \bar{G}^{\wedge}: \chi \equiv 1 \text { on } H\right\}
$$

we have as usual $\bar{H}=\cap\{\operatorname{ker} \chi: \chi \in A\}$ (cf. (2.2)) and hence $H=\cap$ $\{\operatorname{ker}(\chi \mid G): \chi \in A\}$. For $\chi \in \bar{G}^{\wedge}$ we have $\chi \mid G \in\langle G, \mathscr{S}\rangle^{\wedge}$, so for $\chi \in \bar{G}^{\wedge}$ there is $\lambda \in \Lambda$ such that $\chi \mid G \in \mathscr{I}(\lambda)$ and hence $\operatorname{ker}(\chi \mid G)$ is $G$-closed (and hence $\mathscr{T}$-closed). It follows that $H$ is $\mathscr{T}$-closed, as required.

REMARK 4.7. It is shown in [6] that every Abelian group $G$ has a largest totally bounded group topology; this is, in fact, the 
topology induced on $G$ by $\left(G_{d}\right)^{\wedge}=\operatorname{Hom}(G, \mathbf{T})$. It is, accordingly, perhaps tempting to believe that the proof of Theorem 4.6 can be adapted to show that $\mathscr{B}(\mathscr{T})$ has a largest element, namely the topology induced on $G$ by the subgroup of $\left(G_{d}\right)^{\wedge}$ generated by $\cup\left\{\langle G, \mathscr{S}\rangle^{\wedge}: \mathscr{S} \in \mathscr{B}(\mathscr{T})\right\}$. This attempt to strengthen Theorem 4.6 fails. We show in Theorem 7.2 below that (with $\mathscr{T}$ the usual topology for $\mathbf{T})$ for $i=0,1$ there are $\mathscr{S}_{i} \in \mathscr{B}(\mathscr{T})$ and $\chi_{i} \in\left\langle\mathbf{T}, \mathscr{S}_{i}\right\rangle^{\wedge}$ such that $\operatorname{ker}\left(\chi_{0} \cdot \chi_{1}\right)$ is a proper $\mathscr{T}$-dense subgroup of $\mathbf{T}$; since $\chi_{0} \cdot \chi_{1}$ is continuous in any topology for $\mathbf{T}$ containing $\mathscr{S}_{0}$ and $\mathscr{S}_{1}$, no such topology can belong to $\mathscr{B}(\mathscr{T})$.

5. On the existence of totally dense subgroups. We recall from $\S 4$ that a subgroup $H$ of a group $G$ is said to be totally dense (in $G$ ) if, for each closed subgroup $K$ of $G$, the set $H \cap K$ is dense in $K$. It is clear (upon taking $K=G$ ) that every totally dense subgroup is dense. It is clear also that if $H$ is totally dense in $G$ and $K$ is a closed subgroup of $G$, then $H \cap K \supsetneq\{e\}$ provided $K \neq$ $\{e\}$. For $G$ compact, the subgroups $H$ of $G$ with this latter, weaker property are exactly the subgroups of $G$ with no coarser (Hausdorff) topological group topology. That is: A totally bounded group < $\mathscr{H}$, $\mathscr{T}>$ admits no coarser group topology if and only if $H \cap K \supsetneq\{e\}$ for every nondegenerate, closed subgroup $K$ of the Weil completion $\bar{H}$. These "minimal groups" have been studied extensively (see for example [27], [10], [30], [15], [16]). The totally dense subgroups of compact groups are exactly those subgroups which, together with all their Hausdorff quotients, are minimal groups [31], [15].

In this section we establish the existence, under suitable conditions, of totally dense, pseudocompact subgroups.

We first remark that not every compact group has a proper, totally dense subgroup. Let $G$ be a compact torsion group, $x \in G$, $K(x)$ the smallest closed subgroup of $G$ containing $x$, and $H$ a totally dense subgroup of $G$. Since $K(x)$ is finite and $H \cap K(x)$ is dense in $K(x)$ we have $H \cap K(x)=K(x)$ and hence $x \in H$. Thus a compact torsion group has no proper totally dense subgroup. (For a concrete example take $p \in \mathbf{P}, \alpha \geqq \omega$ and $G=(Z(p))^{\alpha}$.) We show now that for connected Abelian groups the situation is quite different.

Lemma 5.1. For $\alpha>\omega$, the group $\mathbf{T}^{\alpha}$ contains a proper, pseudocompact, totally dense subgroup.

Proof. This is essentially a consequence of a result of D. Grant [16]: Denoting by $t \mathbf{T}$ the torsion subgroup of $\mathbf{T}$, the group $(t \mathbf{T})^{\alpha}$ is totally dense in $\mathbf{T}^{\alpha}$. As earlier set

$$
\Sigma=\left\{x \in \mathbf{T}^{\alpha}:\left|\left\{\xi<\alpha: x_{\xi} \neq 1\right\}\right| \leqq \omega\right\}
$$


and let $H$ be the subgroup of $\mathbf{T}^{\alpha}$ generated by $\Sigma \cup(t \mathbf{T})^{\alpha}$. Since $\Sigma$ is pseudocompact (2.11) and dense in $\mathbf{T}^{\alpha}, H$ is pseudocompact; since $(t \mathbf{T})^{\alpha}$ is totally dense in $\mathbf{T}^{\alpha}$ and $(t T)^{\alpha} \subset H \subset \mathbf{T}^{\alpha}, H$ is totally dense in $\mathbf{T}^{\alpha}$. The elements of $H$ are those elements of $\mathbf{T}^{\alpha}$ all of whose coordinates, with at most countably many exceptions, are torsion elements of $\mathbf{T}$; hence $H \subset \mathbf{T}^{\alpha}$ and $H \neq \mathbf{T}^{\alpha}$.

LEMMA 5.2. Let $G$ be a compact, connected, Abelian group with $w(G)=\alpha>\omega$. There is a continuous homomorphism of $G$ onto $\mathbf{T}^{\alpha}$.

Proof. It suffices to show that $\hat{G}$ contains a subgroup $B$ isomorphic to $\left(\mathbf{T}^{\alpha}\right)^{\wedge}$; for then by (2.2) the annihilator $A$ in $G$ of $B$ satisfies $G / A \cong \hat{B} \cong \mathbf{T}^{\alpha}$.

Let $I$ be a maximal independent subset of $\hat{G}, B$ the subgroup of $\hat{G}$ generated by $I$, and $W$ the divisible hull (that is, the injective hull) of $\hat{G}$. Then $B \cong \bigoplus_{i \in I} Z_{i} \cong\left(\mathbf{T}^{I}\right)^{\wedge}$, and since $|\widehat{G}|=\alpha$ it follows from

$$
B \subset \hat{G} \subset W=\bigoplus_{i \in I} Q_{i}
$$

that $\omega \cdot|I| \leqq \alpha \leqq \omega \cdot|I|$ and hence $|I|=\alpha$, as required.

THEOREM 5.3. Let $G$ be a compact, connected, Abelian group with $w(G)=\alpha>\omega$. Then $G$ contains a proper, pseudocompact, totally dense subgroup.

Proof. There are by Lemmas 5.1 and 5.2 a continuous homomorphism $\varphi$ from $G$ onto $\mathbf{T}^{\alpha}$ and a proper, pseudocompact, totally dense subgroup $\widetilde{H}$ of $\mathbf{T}^{\alpha}$. We set $H=\varphi^{-1}(\widetilde{H})$. Then $H$ is pseudocompact by Lemma $4.1(\mathrm{~b})$, proper in $G$ since $\widetilde{H}$ is proper in $\widetilde{G}$, and totally dense in $G$ by Lemma $4.1(\mathrm{c})$.

REMARK 5.4. We showed in Lemma 4.1(c) that if $G$ and $\widetilde{G}$ are compact groups, $\varphi$ a continuous homomorphism from $G$ onto $\widetilde{G}$ and $\widetilde{H}$ a totally dense subgroup of $\widetilde{G}$, then $\varphi^{-1}(\widetilde{H})$ is totally dense in $G$. It is not difficult to show that in this statement the requirement that $G$ is compact can be replaced by the hypothesis that ( $G$ is a Hausdorff group and) $\widetilde{G}$ has the form $\widetilde{G}=G / N$ with $N$ a compact, normal subgroup of $G$ and $\varphi$ the cannonical homomorphism. (Since we do not need this statement, we omit the details of the proof.) We note, however, that in general the inverse image under a continuous homomorphism of a totally dense subgroup need not be totally dense. For a simple example one may take the canonical homomorphism 


$$
\varphi: \mathbf{R} \longrightarrow \mathbf{R} / \mathbf{Z} \cong \mathbf{T}
$$

and the torsion subgroup $t \mathbf{T}$ of $\mathbf{T}$; here $\varphi^{-1}(t \mathbf{T})$, which is the group of rational numbers under addition, is not totally dense in $\mathbf{R}$.

In the paragraph preceding Lemma 5.1 we showed that there are many compact, totally disconnected, Abelian groups $G$, even with $w(G)>\omega$, with no proper, totally dense subgroup. There are, however, compact totally disconnected groups with such a subgroup. The following definition sets forth a useful concept; the positive result appears below in Theorem 5.6.

Definition. Let $G$ be a compact, totally disconnected Abelian group and for $p \in \mathbf{P}$ let $\hat{G}_{p}$ be the $p$-primary subgroup of the dual group $\hat{G}$. Then $p$ is abundant in $G$ if $\left|\widehat{G}_{p}\right|>\omega$.

Lemma 5.5. Let $I$ be an infinite set of primes, $\left\{\alpha_{p}: p \in I\right\} a$ set of cardinal numbers, $G_{p}=(Z(p))^{\alpha_{p}}$ for $p \in I, G=\Pi_{p \in I} G_{p}$ and $H=\bigoplus_{p \in I} G_{p}$. Then $H$ is the smallest totally dense subgroup of $G$ in the following sense:

(a) $H$ is totally dense in $G$, and

(b) if $J$ is a totally dense subgroup of $G$, then $H \subset J$.

Proof. We have noted already that every torsion element of a group belongs to every totally dense subgroup. It is clear in the present case that $H$ is the torsion subgroup of $G$; statement (b) follows.

To prove (a) it is enough to show that for every closed subgroup $K$ of $G$, the torsion subgroup $t K$ of $K$ is dense in $K$ (for, as just indicated, $t K$ is a subgroup of $H$ ). We have from (2.1)

$$
\hat{G} \cong \bigoplus_{p \in I}\left[\bigoplus_{\varepsilon<\alpha_{p}} Z(p)_{\xi}\right],
$$

with $Z(p)_{\xi}=Z(p)$ for $\xi<\alpha_{p}$. It follows from (2.2) that $\hat{K}$ is a quotient of $\hat{G}$. Hence $\hat{K}$ is (isomorphic to) a group of the form $\bigoplus_{p \in I}\left[\bigoplus_{\xi<\alpha_{p}}\left(F(p)_{\xi}\right]\right.$ where, for $\xi<\alpha_{p}, \quad F(p)_{\xi}=\{0\}$ or $F(p)_{\xi}=Z(p)_{\xi}$. Then

$$
K \cong \prod_{p \in I}\left[\prod_{\xi<\alpha_{p}} F(p)_{\xi}\right],
$$

a product of finite cyclic groups. It is then clear that the torsion subgroup of $K$ is dense in $K$, as required.

TheOREM 5.6. Let $G$ be a compact, totally disconnected, Abelian group in which infinitely many primes are abundant. Then $G$ has a proper, pseudocompact, totally dense subgroup. 
Proof. As in the proof of Theorem 4.3 we write $\hat{G}=\bigoplus_{p \in P} \hat{G}_{p}$ with $\hat{G}_{p}$ the $p$-primary subgroup of $\hat{G}$. There is $I \subset P$ with $|I|=\omega$ such that $\left|\widehat{G}_{p}\right|>\omega$ for $p \in I$. We find a subgroup $S_{p}$ of $\hat{G}_{p}$ with $S_{p}=\bigoplus_{\xi<\omega^{+}} Z(p)_{\xi}$ and we set $S=\bigoplus_{p \in I} S_{p}$. From (2.1) we have

$$
\widehat{S}=\prod_{p \in I} \hat{S}_{p}=\prod_{p \in I} \prod_{\xi<\omega^{+}}+Z(p)_{\xi}=\prod_{p \in I}(Z(p))^{\omega^{+}},
$$

and from (2.2) there is a homomorphism $\varphi$ from $G$ onto $\hat{S}$. We set $H=\bigoplus_{p \in I}(Z(p))^{\omega^{+}}$and

$$
\Sigma=\left\{x \in \prod_{p \in I}(Z(p))^{\omega^{+}}:\left|\left\{\langle p, \xi\rangle \in I \times \omega^{+}: x_{p, \xi} \neq 0\right\}\right| \leqq \omega\right\}
$$

and we denote by $\tilde{H}$ the subgroup of $\hat{S}$ generated by $H \cup \Sigma$. Now $H$ is totally dense in $\widehat{S}$ by Lemma 5.5 , and $\Sigma$ is a pseudocompact subgroup of $\hat{S}$ (2.11) dense in $\hat{S}$ and hence in $\widetilde{H}$. It follows that $\widetilde{H}$ is a pseudocompact, totally dense subgroup of $\hat{S}$. It is clear further that $\widetilde{H}$ is a proper subset of $\hat{S}$. It follows then from Lemma 4.1 that $\varphi^{-1}(\tilde{H})$ is a proper, pseudocompact, totally dense subgroup of $G$, as required.

6. On the cardinality of totally dense subgroups. We have described several groups $G$ in which it is possible to find a proper, totally dense subgroup $H$; here we investigate the question whether $H$ may be chosen so that in addition $|H|$ is small relative to $|G|$.

Our interest in this general question arose when we recalled the so-called Hewitt-Marczewski-Pondiczery phenomenon (see for example [11] (Problem 2L) or [3] (Corollary 3.18)): for $\alpha \geqq \omega$, a product of $\leqq 2^{\alpha}$ spaces with density character $\leqq \alpha$ itself has density character $\leqq \alpha$. Thus for example the group $\mathbf{T}^{2^{\alpha}}$ has a dense subset $D$ with $|D| \leqq \alpha$; clearly the subgroup $H$ generated by $D$ satisfies $|H| \leqq \alpha$. May $H$ be taken totally dense? Are there models of ZFC in which some totally dense subgroup $H$ of $\mathbf{T}^{2^{\alpha}}$ satisfies $|H|<$ $2^{\alpha}$ ? $|H|<2^{2^{\alpha}}$ ? It is questions like these which motivated the present section.

THEOREM 6.1. Let $G$ be a locally compact, $\sigma$-compact group and $H$ a totally dense subgroup. Then $|H| \geqq w(G)$ (and hence $\left.|G| \leqq 2^{|H|}\right)$.

Proof. If $G$ is discrete we have $w(G)=|G| \leqq \omega$ and $H=G$. We assume that $G$ is not discrete, we denote by $\kappa$ the local weight of $G$ at $e$, for $x \in H$ with $x \neq e$ we choose by (2.6) a compact, $G_{\delta}$ subgroup $H(x)$ of $G$ with $x \notin H(x)$, and we set

$$
K=\cap\{H(x): x \in H, x \neq e\} .
$$


Since $K$ is a closed subgroup of $G$ and $K \cap H=\{e\}$ we have $K=\{e\}$. Denoting by $\psi(G, e)$ the pseudoweight of $G$ at $e$-that is, the minimal number of open subsets of $G$ whose intersection is equal to $\{e\}-$ we have

$$
\psi(G, e) \leqq \omega \cdot|H|=|H|
$$

and also (since $G$ is locally compact) $\psi(G, e)=\kappa$. Then from (2.4) we have

$$
w(G)=\kappa \leqq|H| \text { and }|G|=2^{\kappa} \leqq 2^{|H|},
$$

as required.

For a compact group assumed Abelian, a stronger lower bound (on the cardinality of a totally dense subgroup) is available. It is convenient to separate our treatment of the connected and the totally disconnected cases; these are combined in Corollary 6.5.

LEMMA 6.2. Let $G$ and $\widetilde{G}$ be topological groups, $\Phi$ a continuous homomorphism from $G$ onto $\widetilde{G}, H \subset G$ and $\widetilde{H}=\varphi[H]$. If $H$ is totally dense in $G$, then $\widetilde{H}$ is totally dense in $\widetilde{G}$.

Proof. Let $\widetilde{K}$ be a closed subgroup of $\widetilde{G}$ and set $K=\varphi^{-1}(\widetilde{K})$; then $K$ is a closed subgroup of $G$. Since $H$ is totally dense in $G$ the set $H \cap K$ is dense in $K$ and hence $\varphi[H \cap K]$, which is $\widetilde{H} \cap \widetilde{K}$, is dense in $\varphi[K]=\widetilde{K}$.

THEOREM 6.3. Let $G$ be a compact, connected, Abelian group with $w(G)=\alpha>\omega$ and let $H$ be a totally dense subgroup of $G$. Then $|H|=|G|$.

Proof. There is by Lemma 5.2 a continuous homomorphism $\varphi$ from $G$ onto $T^{\alpha}$. The two-element group $\{+1,-1\}$ is a (torsion) subgroup of $\mathbf{T}$ and $\{+1,-1\}^{\alpha}$ is a torsion subgroup of $\mathbf{T}^{\alpha}$. Since $\varphi[H]$ is (by Lemma 6.2) a totally dense subgroup of $\mathbf{T}^{\alpha}$ and every such subgroup contains all torsion elements, we have

$$
|H| \geqq|\varphi[H]| \geqq|\{+1,-1\}|^{\alpha}=2^{\alpha}=|G|,
$$

as required.

THEOREM 6.4. Let $G$ be a compact, totally disconnected Abelian group and $H$ a totally dense subgroup with $|H|=\alpha$. Then $|G| \leqq \alpha^{\prime \omega}$.

Proof. If $|G|<\omega$ then $G=H$. If $\omega \leqq|G| \leqq 2^{\omega}$ then since $|G|=2^{\omega(G)}$ we have $|G|=2^{\omega}$ and from the fact that $H$ is dense in $G$ 
it follows that $\alpha \geqq \omega$ and hence $|G|=\alpha^{\omega}$. We assume in what follows that $|G|>2^{\omega}$, we note from $|G|=2^{|\hat{G}|}$ that $|\hat{G}|>\omega$, and using (2.12) we write $\hat{G}=\bigoplus_{p \in \mathbf{P}} \widehat{G}_{p}$ with $\widehat{G}_{p}$ the $p$-primary subgroup of $\hat{G}$. We set

$$
I=\left\{p \in \mathbf{P}:\left|\widehat{G}_{p}\right|>\omega\right\},
$$

we note $|I| \neq \phi$, and for $p \in I$ we denote by $S_{p}$ the socle

$$
S_{p}=\left\{\chi \in \hat{G}_{p}: \text { order of } \chi=p\right\} \cup\{e\} .
$$

We set $\alpha_{p}=\left|S_{p}\right|$ and we note from (2.14) that $\alpha_{p}=\left|\widehat{G}_{p}\right|$ for $p \in I$.

Now let $S=\bigoplus_{p \in I} S_{p}$. For $p \in I$ we have $S_{p}=\bigoplus_{\xi<\alpha_{p}} Z(p)_{\xi}$ and hence $\widehat{S}_{p}=(Z(p))^{\alpha}{ }_{p}$. Since $\hat{S}=\prod_{p \in I} \hat{S}_{p}$ we have

$$
|\widehat{S}|=\prod_{p \in I}\left|\hat{S}_{p}\right|=\prod_{p \in I} 2^{\alpha_{p}} \text {. }
$$

We claim further that $|\hat{S}|=|G|$. Indeed

$$
\begin{aligned}
w G & =|\hat{G}|=\left|\bigoplus_{p \in \mathbf{P}} \hat{G}_{p}\right|=\left|\left(\bigoplus_{p} \bigoplus_{\mathbf{P} \backslash I} \hat{G}_{p}\right) \bigoplus\left(\bigoplus_{p \in I} \hat{G}_{p}\right)\right| \\
& =\omega \cdot\left|\bigoplus_{p \in I} \widehat{G}_{p}\right|=\sum_{p \in I}\left|\hat{G}_{p}\right|=\sum_{p \in I} \alpha_{p} \\
& =\left|\bigoplus_{p \in I} S_{p}\right|=|S|=\left|(\hat{S})^{\wedge}\right|=w(\hat{S})
\end{aligned}
$$

and hence $|G|=2^{w G}=2^{w(\hat{S})}=|\hat{S}|$.

There is by (2.2) a homomorphism $\varphi$ from $G$ onto $\widehat{S}$. Since $\varphi[H]$ is totally dense in $\hat{S}$ (by Lemma 6.2), the set $\varphi[H]$ contains the torsion subgroup of

$$
\widehat{S}=\prod_{p \in I} \hat{S}_{p}=\prod_{p \in I}(Z(p))^{\alpha_{p}} ;
$$

that is, $\varphi[H] \supset \bigoplus_{p \in I}(Z(p))^{\alpha_{p}}$. It follows that

$$
\alpha=|H| \geqq|\varphi[H]| \geqq\left|(Z(p))^{\alpha_{p}}\right|=2^{\alpha_{p}} \quad \text { for } \quad p \in I
$$

and hence

$$
|G|=|\widehat{S}|=\prod_{p \in I} 2^{\alpha_{p}} \leqq \alpha^{|I|} \leqq \alpha^{\omega},
$$

as required.

CoRollary 6.5. Let $G$ be a compact Abelian group and $H$ a totally dense subgroup with $|H|=\alpha$. Then $|G| \leqq \alpha^{\omega}$.

Proof. If $|G| \leqq 2^{\omega}$ then as in the first paragraph of the proof of Theorem 6.4 we have either $|G|=\alpha$ or $|G|=\alpha^{\omega}$. We assume in what follows that $|G|>2^{\omega}$. 
Let $C$ be the component of the identity in $G$ and $\varphi$ the canonical homomorphism of $G$ onto the totally disconnected group $G / C$. If $|C|=|G|$ then from $|C|=2^{w C}$ we have $w C>\omega$ and since $H \cap C$ is totally dense in $C$ we have

$$
|G|=|C|=|H \cap C| \leqq|H|=\alpha \leqq \alpha^{\omega}
$$

from Theorem 6.3 (applied to $C$ ); and if $|C|<|G|$ then since $\varphi[H]$ is totally dense in $G / C$ we have

$$
|G|=|G / C| \leqq|\varphi[H]|^{\omega} \leqq \alpha^{\omega}
$$

from Theorem 6.4. The proof is complete.

The general conclusion to be drawn from the results above in this section is that a totally dense subgroup $H$ of a compact Abelian group $G$ cannot be much smaller (in cardinality) than $G$ itself. It becomes reasonable to ask: is $|H|<|G|$ possible? The answer here is "Yes": take $G=\prod_{p \in \mathbf{P}} Z(p)$ and $H=\bigoplus_{p \in \mathbf{P}} Z(p)$. More generally, we have this result.

THEOREM 6.6. For every infinite cardinal $\bar{\alpha}$ there are a compact, totally disconnected Abelian group $G$ with $|G|>\bar{\alpha}$ and a totally dense subgroup $H$ of $G$ such that $|H|<|G|$.

Proof. We recall from Lemma 5.5 that if $\left\{\alpha_{p}: p \in \mathbf{P}\right\}$ is a set of cardinal numbers, $G_{p}=(Z(p))^{\alpha}{ }^{\alpha}$ for $p \in \mathbf{P}$ and $G=\prod_{p \in \mathbf{P}} G_{p}$ and $H=\bigoplus_{p \in \mathrm{P}} G_{p}$, then $H$ is totally dense in $G$. In this case, writing $\alpha=\sum_{p} \alpha_{p}$ and $\gamma=\sum_{p} 2^{\alpha_{p}}$, we evidently have $|H|=\gamma$ and

$$
|G|=\prod_{p}\left|G_{p}\right|=\prod_{p} 2^{\alpha_{p}}=2^{\sum_{p}^{\alpha} \alpha_{p}}=2^{\alpha} .
$$

(It follows from Theorem 6.4 that $2^{\alpha} \leqq|H|^{\omega}=\gamma^{\omega}$. In the present specialized context this is easily verified directly: We have $2^{\alpha_{p}} \leqq \gamma$ for $p \in \mathbf{P}$, and hence

$$
2^{\alpha}=\prod_{p} 2^{\alpha_{p}} \leqq \gamma^{|\mathbf{P}|}=\gamma^{\omega} .
$$

To arrange $|H|<|G|$, we recall the classical theorem of J. König (see for example [1], or [3] (1.19)): If $\left\{a_{i}: i \in I\right\}$ and $\left\{b_{i}: i \in I\right\}$ are sets of cardinal numbers with $a_{i}<b_{i}$ for all $i \in I$, then $\sum_{i \in I} a_{i}<$ $\prod_{i \in I} b_{i}$. In the present context, where $|H|=\sum_{p} 2^{\alpha_{p}}$ and $|G|=\Pi_{p} 2^{\alpha_{p}}$, it is enough, denoting by $p^{\prime}$ the successor of $p$ in $\mathbf{P}$, to have $\alpha_{2}=\bar{\alpha}$ and $2^{\alpha_{p}}<2^{\alpha_{p^{\prime}}}$ for $p \in \mathbf{P}$ (for example, $\alpha_{p^{\prime}}=2^{\alpha_{p}}$ ). The proof is complete.

Let us notice at this point, following Corson [9], that the subgroup $H$ of $G$ in the proof of Theorem 6.6 is $\sigma$-compact and hence 
Lindelöf. (The proof is not difficult: The group $G$ is naturally identified with a product $\Pi_{\xi<\alpha} F_{\xi}$ with $\alpha=\sum_{p \in \mathbf{P}} \alpha_{p}$ and with $F_{\xi}$ a finite group for $\xi<\alpha$; writing

$$
H_{n}=\left\{x \in H:\left|\left\{\xi<\alpha: x_{\xi} \neq e_{\xi}\right\}\right| \leqq n\right\} \text { for } n<\omega
$$

we see that each set $H_{n}$ is closed in $G$ and hence compact, and that $H=\bigcup_{n<\omega} H_{n}$.) Since $H$ is dense and proper in $G$ it is not pseudocompact, for a pseudocompact, Lindelöf space is compact. In an early version of this manuscript the authors, motivated by these observations, raised (but could not solve) the following question: Are there a compact, Abelian group $G$ and a totally dense, pseudocompact subgroup $H$ such that $|H|<|G|$ ? Subsequently the firstlisted author and Lewis C. Robertson have shown that this question is undecidable on the basis of the usual axioms of Zermelo-Fraenkel set theory. A precise statement will appear, together with related results, in [5].

While it is not difficult to find pseudocompact groups that are not countably compact (to the best of our knowledge it was Kister [22] who first explicitly considered this question), the $\Sigma$-products defined above and used in various connections are in fact countably compact; indeed they have the stronger property that each of their countable subsets has compact closure. In view then of the ready availability of proper, dense, countably compact subgroups of (many) compact groups, it is perhaps reasonable to ask a "strengthened" form of the question above: Are there a compact (Abelian) group $G$ and a totally dense, countably compact subgroup $H$ such that $|H|<|G|$ ? We now answer that question in the negative.

THEOREM 6.7. Let $G$ be a compact, totally disconnected group and $H$ a totally dense, countably compact subgroup. Then $H=G$.

Proof. It follows from [18] (Theorem 25.16) that for $x \in G$ there is a compact, metric subgroup $H(x)$ of $G$ such that $x \in H(x)$. (This observation and many related results about "metric elements" are given in [34].) Since $H \cap H(x)$ is dense in $H(x)$ (and closed), we have $H \cap H(x)=H(x)$ and hence $x \in H(x) \subset H$.

COROLlARy 6.8. Let $G$ be a compact Abelian group and $H$ a totally dense, countably compact subgroup. Then $|H|=|G|$.

Proof. If $w G \leqq \omega$ then $G$ is metrizable and $H$ is a compact metric space; since $H$ is dense in $G$ we have in fact $H=G$. We assume in what follows that $w G>\omega$.

Let $C$ be the component of the identity in $G$ and $\rho$ the canoni- 
cal homomorphism of $G$ onto the totally disconnected group $G / C$. If $|C|=|G|$ then since $H \cap C$ is totally dense in $C$ and $w C>\omega$ we have from Theorem 6.3 that

$$
|G| \geqq|H| \geqq|H \cap C|=|C|=|G| .
$$

If $|C|<|G|$ then since $\varphi[H]$ is a totally dense, countably compact subgroup of $G / C$ we have from Theorem 6.7 that $\varphi[H]=G / C$ and hence

$$
|G| \geqq|H| \geqq|\varphi[H]|=|G / C|=|G| \text {. }
$$

REMARK 6.9. In the interest of completeness we cite a result of $\mathrm{H}$. Wilcox [34] related to those of this section but formally neither weaker nor stronger than ours. Suppose that $G$ is an infinite, compact Abelian group for which $w G$ has the form $w G=2^{\alpha}$ (so $|G|=2^{2^{\alpha}}$ ) with $\alpha^{\omega}<2^{\alpha}$. Then for every dense, pseudocompact subgroup $H$ of $G$ with $|H| \leqq \alpha^{\omega}$ there is an infinite, closed subgroup $K$ of $G$ such that $H \cap K=\{e\}$ ([34](Theorem 2.2)). In particular, $H$ is not totally dense in $G$. We note from Corollary 6.5 above that for such a group $G$ any totally dense subgroup $H$, whether pseudocompact or not, must in fact satisfy $|H|>2^{\alpha}$ (since from $|H| \leqq 2^{\alpha}$ would follow the contradiction

$$
\left.2^{2^{\alpha}}=|G| \leqq|H|^{\omega} \leqq\left(2^{\alpha}\right)^{\omega}=2^{\alpha}\right) .
$$

7. Some totally bounded topologies on $\mathbf{T}$. We have noted already that every pseudocompact group topology is totally bounded and we have shown that no pseudocompact group topology properly extending a compact group topology can have the same family of closed subgroups. We show in this section that there exist many totally bounded topologies for $\mathbf{T}$ with the same closed subgroups; our construction allows us to deduce, somewhat unexpectedly (cf. Remark 4.7), that there is no largest such topology.

For $p \in \mathbf{P}$ we use the symbol $Z\left(p^{\infty}\right)$ as usual and we recall the isomorphism $\mathbf{T} \simeq\left(\bigoplus_{p \in \mathbf{P}} Z\left(p^{\infty}\right)\right) \oplus \mathbf{R}$ (cf. [18] (A.14) or [12] (23.1)). When convenient we identify $Z\left(p^{\infty}\right)$ and $\mathbf{R}$ with their natural copies inside $\mathbf{T}$ (that is, we write $Z\left(p^{\infty}\right) \subset \mathbf{T}$ and $\mathbf{R} \subset \mathbf{T}$ ) and we write $\mathbf{T}$ additively, using the symbol 0 to denote the identity of $\mathbf{T}$ (or of any of the groups $Z\left(p^{\infty}\right)$, or of $\left.\mathbf{R}\right)$.

Lemma 7.1. There is a homomorphism $f$ of $\mathbf{T}$ into $\mathbf{T}$ such that for all $m, n \in \mathbf{Z}$ with $\langle m, n\rangle \neq\langle 0,0\rangle$ the set $\{x \in \mathbf{T}: m x+n f(x)=0\}$ is finite.

Proof. For $p \in \mathbf{P}$ let $i_{p}$ denote the identity function on $Z\left(p^{\infty}\right)$; 
that is, $i_{p}(x)=x$ for $x \in Z\left(p^{\infty}\right)$. The endomorphism group Hom $\left(Z\left(p^{\infty}\right)\right.$, $Z\left(p^{\infty}\right)$ ) has been analyzed in detail (cf. [18] (p. 181) or [12] (25.2)); we here need only that its cardinality is $2^{\omega}$, so that we may choose an endomorphism $g_{p}$ of $Z\left(p^{\infty}\right)$ such that $i_{p}$ and $g_{p}$ are independent. That is: for $a, b \in \mathbf{Z}$, the endomorphism $a g_{p}+b i_{p}$ is the zero function only when $a=b=0$. (We remark for later use that in fact for $p \in \mathbf{P}$ there is an independent subset $B(p)$ of Hom $\left(Z\left(p^{\infty}\right), Z\left(p^{\infty}\right)\right)$ with $i_{p} \in B(p)$ and with $|B(p)|=2^{\omega}$.)

Let [ ] denote the usual "greatest integer" function. We set

$$
f_{p}=p \cdot g_{p}+\left[p^{1 / 4}\right] \cdot i_{p} \text { for } p \in \mathbf{P},
$$

we define $f_{\mathbf{R}} \in \operatorname{Hom}(\mathbf{R}, \mathbf{R})$ by the rule $f_{\mathbf{R}}(x)=\sqrt{2} x$, and we define

$$
f=\left(\bigoplus_{p} f_{p}\right) \oplus f_{\mathbf{R}}
$$

that is, we choose $f \in \operatorname{Hom}(\mathbf{T})$ so that $f \mid Z\left(p^{\infty}\right)=f_{p}$ and $f \mid \mathbf{R}=f_{\mathbf{R}}$. It remains to show $f$ is as required.

We fix $m, n \in \mathbf{Z}$ with $\langle m, n\rangle \neq\langle 0,0\rangle$ and we set

$$
\begin{aligned}
& K=\{x \in \mathbf{T}: m x+n f(x)=0\}, \\
& K(p)=\left\{x \in Z\left(p^{\infty}\right): m x+n f(x)=0\right\} \text { for } p \in \mathbf{P}, \text { and } \\
& K(\mathbf{R})=\{x \in \mathbf{R}: m x+n f(x)=0\} .
\end{aligned}
$$

It is clear that $K=\left(\bigoplus_{p} K(p)\right) \oplus K(\mathbf{R})$. Thus to show $|K|<\omega$ it is enough to show the following three statements.

(a) $|K(\mathbf{R})|<\omega$;

(b) $|K(p)|<\omega$ for all $p \in \mathbf{P}$;

(c) there is $N<\omega$ such that $K(p)=\{0\}$ for $N<p \in \mathbf{P}$.

It is clear in any event that if $n=0$ then $K=\{x \in \mathbf{T}: m x=0\}$ and hence $K$ is finite. We assume in what follows that $n \neq 0$.

(a) For $x \in K(\mathbf{R})$ we have $m x+n \sqrt{2} x=0$ and hence $x=0$; thus in fact $K(\mathbf{R})=\{0\}$.

(b) If there is $p \in \mathbf{P}$ with $|K(p)|=\omega$ then since every proper subgroup of $Z\left(p^{\infty}\right)$ is finite we have $K(p)=Z\left(p^{\infty}\right)$ and the function

$$
x \longrightarrow m x+n f(x)=n p g_{p}(x)+\left(m+n\left[p^{1 / 4}\right]\right) x
$$

is the zero function on $Z\left(p^{\infty}\right)$. It follows that $n p=0$, contrary to the fact $n \neq 0, p \in \mathbf{P}$.

(c) Let $N=\max \left\{16,(m+1)^{4}, n^{4}\right\}$ and $N<p \in \mathbf{P}$. Since $p>16$ we have

$$
p^{1 / 4}\left(1+p^{1 / 4}\right)<p^{1 / 4}\left(2 p^{1 / 4}\right)<p^{1 / 4} \cdot p^{1 / 2}=p^{3 / 4}<p ;
$$

since $p>(m+1)^{4}$ we have $m+n\left[p^{1 / 4}\right] \neq 0$ (otherwise $1 \leqq\left[p^{1 / 4}\right]$ and $\left[p^{1 / 4}\right] \mid m$, a contradiction). 
Let $a$ be an element of $Z\left(p^{\infty}\right)$ such that $a \neq 0$ and $p a=0$. Since $a$ belongs to every proper subgroup of $Z\left(p^{\infty}\right)$, it is enough (to show $K(p)=\{0\})$ to show $a \notin K(p)$. If $a \in K(p)$ then from $p a=0$ we have $p g_{p}(a)=0$ and hence

$$
\begin{aligned}
0 & =m a+n f(a)=n p g_{p}(a)+\left(m+n\left[p^{1 / 4}\right]\right) a \\
& =\left(m+n\left[p^{1 / 4}\right]\right) a
\end{aligned}
$$

and hence $p \mid\left(m+n\left[p^{1 / 4}\right]\right)$. From $\left(m+n\left[p^{1 / 4}\right]\right) \neq 0$ it follows that

$$
\begin{aligned}
p & \leqq\left|m+n\left[p^{1 / 4}\right]\right| \leqq|m|+|n|\left[p^{1 / 4}\right] \\
& <p^{1 / 4}+p^{1 / 4}\left[p^{1 / 4}\right] \leqq p^{1 / 4}\left(1+p^{1 / 4}\right)<p,
\end{aligned}
$$

a contradiction. The proof is complete.

For two topologies $\mathscr{T}^{\prime}, \mathscr{T}^{\prime \prime}$ on a set $X$ we denote by $\mathscr{T}^{\prime} \mathrm{V}$ $\mathscr{T}^{\prime \prime}$ the least (the smallest) topology on $X$ containing $\mathscr{T}^{\prime}$ and $\mathscr{T}^{\prime \prime}$.

THEOREM 7.2. Let $\mathscr{T}$ be the usual topology for $\mathbf{T}$. There is a set $\left\{\mathscr{T}_{\xi}: \xi<2^{\omega}\right\}$ of topological group topologies for $\mathbf{T}$ such that

(a) $\mathscr{T} \varsubsetneqq \mathscr{T}_{\xi}$ for $\xi<2^{\omega}$;

(b) $\left\langle T, \mathscr{T}_{\xi}\right\rangle$ is totally bounded for $\xi<2^{\omega}$;

(c) $\mathscr{T}$ and $\mathscr{T}_{\xi}$ have the same closed subgroups;

(d) every continuous homomorphism $h:\left\langle\mathbf{T}, \mathscr{T}_{\xi}\right\rangle \rightarrow\langle\mathbf{T}, \mathscr{T}\rangle$ satisfies $|h[\mathbf{T}]|=1$ or $h[\mathbf{T}]=\mathbf{T} ;$ and

(e) for $\xi<\xi^{\prime}<2^{\omega}$ there is a proper, $\mathscr{T}$-dense, $\left(\mathscr{T}_{\xi} \vee \mathscr{T}_{\xi^{\prime}}\right.$ )closed subgroup of $\mathbf{T}$.

Proof. For $p \in \mathbf{P}$ with $p>2$ we choose and fix $g_{p} \in \operatorname{Hom}\left(Z\left(p^{\infty}\right)\right)$, $Z\left(p^{\infty}\right)$ ) as in (the proof of) Theorem 7.1 and for $\xi<2^{\omega}$ we choose $g_{2}^{f} \in \operatorname{Hom}\left(Z\left(2^{\infty}\right), Z\left(2^{\infty}\right)\right)$ so that $\left\{g_{2}^{\xi}: \xi<2^{\omega}\right\} \cup\left\{i_{2}\right\}$ is independent. We define

$$
\begin{aligned}
& f_{p}^{\xi}=f_{p}=p \cdot g_{p}+\left[p^{1 / 4}\right] \cdot i_{p} \text { for } 2<p \in \mathbf{P}, \xi<2^{\omega}, \\
& f_{2}^{\xi}=2 \cdot g_{2}^{\hat{\xi}}+\left[2^{1 / 4}\right] \cdot i_{2} \text { for } \xi<2^{\omega}, \\
& f_{\mathbf{R}}^{\xi}=f_{\mathbf{R}}=\sqrt{\overline{2}} \cdot i_{\mathbf{R}} \text { for } \xi<2^{\omega},
\end{aligned}
$$

and we define $f^{\xi} \in \operatorname{Hom}(\mathbf{T}, \mathbf{T})$ so that $f^{\xi} \mid Z\left(p^{\infty}\right)=f_{p}^{\xi}$ for $p \in \mathbf{P}$ and $f^{\xi} \mid \mathbf{R}=f_{\mathbf{R}}^{\xi}$. We note that each $f^{\xi}$ is a homomorphism as in (the statement of) Lemma 7.1.

For $\xi<2^{\omega}$ let $\mathscr{H}(\xi)$ be the subgroup of $\operatorname{Hom}(\mathbf{T}, \mathbf{T})$ generated by $\langle\mathbf{T}, \mathscr{T}\rangle^{\wedge} \cup\left\{f^{\xi}\right\}$ and let $\mathscr{T}_{\xi}$ be the topology induced on $\mathbf{T}$ by $\mathscr{H}(\xi)$.

(a) Since $\langle\mathbf{T}, \mathscr{T}\rangle^{\wedge} \subset \mathscr{H}(\xi)$ we have $\mathscr{T} \subset \mathscr{T}_{\xi}$, and since $f^{\xi}$ is $\mathscr{T}_{\xi}$-continuous but not $\mathscr{T}$-continuous we have $\mathscr{T} \neq \mathscr{T}_{\xi}$.

(b) This follows from (2.8). 
(c) Let $H$ be a $\mathscr{T}_{\xi}$-closed subgroup of $\mathbf{T}$, and let $\overline{\mathbf{T}}_{\xi}$ denote the Weil completion of $\left\langle\mathbf{T}, \mathscr{T}_{\xi}\right\rangle$ and $\bar{H}$ the closure in $\overline{\mathbf{T}}_{\xi}$ of $H$. For $h \in \mathscr{P}(\xi)$ let $\bar{h}$ denote the continuous extension to $\overline{\mathbf{T}}_{\xi}$ of $h$, and (retaining additive notation) set

$$
A=\{h \in \mathscr{H}(\xi): \bar{h} \equiv 0 \text { on } H\} .
$$

From (2.8) it follows $\mathscr{\mathscr { C }}(\xi)=\left\langle\mathbf{T}, \mathscr{T}_{\xi}\right\rangle^{\wedge}$ and hence $\{\bar{h}: h \in \mathscr{H}(\xi)\}=\overline{\mathbf{T}}_{\xi}$. Since

$$
\vec{H}=\cap\{\operatorname{ker}(\bar{h}): h \in A\}
$$

we have $H=\cap\{\operatorname{ker}(h): h \in A\}$. For every $h \in A \subset \mathscr{H}(\xi)$ there are $m, n \in \mathbf{Z}$ with $h=m i+n f^{\xi}$, so from Lemma 7.1 it follows that $\operatorname{ker}(h)=\mathbf{T}$ or $\operatorname{ker}(h)$ is finite. It follows that each $\operatorname{ker}(h)$ with $h \in A$, and hence $H$ itself, is $\mathscr{T}$-closed.

(d) Let $h=m i+n f^{\xi} \in\left\langle\mathbf{T}, \mathscr{T}_{\xi}\right\rangle^{\wedge}$. If $\langle m, n\rangle=\langle 0,0\rangle$ then $|h[\mathbf{T}]|=$ 1. If $\langle m, n\rangle \neq\langle 0,0\rangle$ then $h[\mathbf{R}]=\mathbf{R}$ and for $p \in \mathbf{P}$ from $h\left[Z\left(p^{\infty}\right)\right] \cong$ $Z\left(p^{\infty}\right) / \operatorname{ker}\left(h \mid Z\left(p^{\infty}\right)\right)$ and $|\operatorname{ker}(h)|<\omega$ we have $\left|h\left[Z\left(p^{\infty}\right)\right]\right|=\omega$ and hence $h\left[Z\left(p^{\infty}\right)\right]=Z\left(p^{\infty}\right)$. It follows that $h[\mathbf{T}]=\mathbf{T}$.

(e) For $\xi<\xi^{\prime}<2^{\omega}$ the functions $g_{2}^{\xi}$ and $g_{2}^{\hat{f}^{\prime}}$ are independent; in particular there is $\bar{x} \in Z\left(2^{\infty}\right)$ such that

$$
\left(2 g_{2}^{\hat{乏}}-2 g_{2}^{\hat{\xi}^{\prime}}\right)(\bar{x}) \neq 0
$$

and hence $\left(f^{\hat{s}}-f^{\hat{s}^{\prime}}\right)(\bar{x}) \neq 0$. But for $2<p \in \mathbf{P}$ we have

$$
f_{p}^{\xi}=f_{p}^{\xi^{\prime}}=f_{p}
$$

and hence

$$
\left(f^{\xi}-f^{\xi^{\prime}}\right)(x)=f_{p}(x)-f_{p}(x)=0 \text { for } x \in Z\left(p^{\infty}\right) .
$$

Thus $f^{\xi}-f^{\xi^{\prime}}$ is a $\left(\mathscr{T}_{\xi} \vee \mathscr{T}_{\xi^{\prime}}\right)$-continuous homomorphism whose kernel is proper in $\mathbf{T}$ and contains $Z\left(p^{\infty}\right)$ for all $p \in \mathbf{P}$ with $p>2$. The set $\operatorname{ker}\left(f^{\xi}-f^{\xi^{\prime}}\right)$ is then a subgroup of $\mathbf{T}$ as required in (e).

The proof is complete.

\section{REFERENCES}

1. Heinz Bachmann, Transfinite Zahlen, Ergebnisse der Mathematik and ihrer Grenzgebiete vol. 1, Springer-Verlag, Berlin-Heidelberg-New York, 1967.

2. W. W. Comfort and G. L. Itzkowitz, Density character in topological groups, Math. Annalen, 226 (1977), 223-227.

3. W. W. Comfort and S. Negrepontis, The Theory of Ultrafilters, Grundlehren der math. Wissenschaften vol. 211, Springer-Verlag, Berlin-Heidelberg-New York, 1974.

4. W. W. Comfort and Lewis C. Robertson, Proper pseudocompact extensions of compact Abelian group topologies, Proc. Amer. Math. Soc., to appear.

5. - Cardinality constraints for pseudocompact and for totally dense subgroups of compact Abelian groups, manuscript in preparation. 
6. W. W. Comfort and K. A. Ross, Topologies induced by groups of characters, Fundamenta Math., 55 (1964), 283-291.

7. — Pseudocompactness and uniform continuity in topological groups, Pacific J. Math., 16 (1966), 483-496.

8. W. W. Comfort and T. Soundararajan, Pseudocompact topological group topologies, Abstracts Amer. Math. Soc., 2 (1981), 87 [Abstract 783-22-5].

9. H. H. Corson, Normality in subsets of product spaces, American J. Math., 81 (1959), 785-796.

10. Doïtchin Doïtchinov, Produits de groups topologiques minimaux, Bull. Sci. Math 2e série, 96 (1972), 59-64.

11. R. Engelking, Outline of General Topology, North-Holland Publ. Co., Amsterdam and John Wiley and Sons, Inc., New York, 1968.

12. László Fuchs, Infinite Abelian Groups Volume I, Pure and Applied Mathematics Vol. 36, Academic Press, New York and London, 1970.

13. Leonard Gillman and Meyer Jerison, Rings of Continuous Functions, D. Van Nostrand Co., Inc., Princeton, N. J., 1960.

14. Irving Glicksberg, Stone-Čech compactifications of products, Trans. Amer. Math. Soc., 90 (1959), 369-382.

15. Douglass L. Grant, Topological groups which satisfy an open mapping theorem, Pacific J. Math., 68 (1977), 411-423.

16. - Arbitrary powers of the roots of unity are minimal Hausdorff topological groups, Topology Proceedings, 4 (1979), 103-108.

17. Edwin Hewitt, Rings of real-valued continuous functions I, Trans. Amer. Math. Soc., 64 (1948), 45-99.

18. Edwin Hewitt and Kenneth A. Ross, Abstract Harmonic Analysis I, Grundlehren der math. Wissenschaften vol. 115, Springer-Verlag, Berlin-Göttingen-Heidelberg, 1963.

19. Abstract Harmonic Analysis II, Grundlehren der math. Wissenschaften vol. 152, Springer-Verlag, New York-Heidelberg-Berlin, 1970.

20. Gerald L. Itzkowitz, Extension of Haar measure for compact connected Abelian groups, Bull. Amer. Math. Soc., 71 (1965), 152-156.

21. S. Janakiraman and T. Soundararajan, Totally bounded topological groups, Pacific J. Math., to appear.

22. J. M. Kister, Uniform continuity and compactness in topological groups, Proc. Amer. Math. Soc., 13 (1962), 37-40.

23. M. Rajagopalan, Topologies in locally compact groups, Math. Annalen, 176 (1968), 169-180.

24. N. W. Rickert, Locally compact topologies for groups, Trans. Amer. Math. Soc., 126 (1967), 225-235.

25. K. A. Ross, Closed subgroups of locally compact Abelian groups, Fundamenta Math., 56 (1965), 241-244.

26. T. Soundararajan, Galois theory for general extension fields, Crelle J., 241 (1970), $49-63$.

27. - Totally dense subgroups of topological groups, pp. 299-300 in: General Topology and Its Relations to Modern Analysis and Algebra III, Proc. 1968 Kanpur Topological Conference, Academia, Prague, 1971.

28. - Cohomology of Galois extentions, J. Pure and Applied Algebra, 11 (1977), $139-150$.

29. - Pseudocompact subgroups of Galois groups, to appear.

30. R. M. Stephenson, Jr., Minimal topological groups, Math. Annalen, 192 (1971), 193-195.

31. L. J. Sulley, $A$ note on $B$ - and $B_{r}$-complete topological groups, Proc. Cambridge Phil. Soc., 66 (1969), 275-279.

32. André Weil, Sur les espaces à structure uniforme et sur la topologie générale, Publ. Math. Univ. Strasbourg, Hermann \& Cie., Paris, 1937. 
33. Howard J. Wilcox, Dense subgroups of compact groups, Proc. Amer. Math. Soc., 28 (1971), 578-580.

34. - Pseudocompact groups, Pacific J. Math., 19 (1966), 365-379.

Received February 18, 1981 and in revised form May 27, 1981.

WESLEYAN UNIVERSITY

MIDDLETOWN, CT 06457

U.S.A.

AND

MADURAI KaMaRAJ UNIVERSITY

MADURAI 625021

INDIA 


\section{PACIFIC JOURNAL OF MATHEMATICS}

\section{EDITORS}

DONALD BABBITT (Managing Editor)

University of California

Los Angeles, California 90024

Hugo RossI

University of Utah

Salt Lake City, UT 84112

C. C. MOORE and ARTHUR Agus

University of California

Berkeley, CA 94720

\section{J. DUGUNDJI}

Department of Mathematics University of Southern California Los Angeles, California 90007

R. FinN and J. MILGRAM Stanford University Stanford, California 94305

\section{ASSOCIATE EDITORS}
R. ARNES
E. F. BeCKENBACH
B. H. Neumann
F. WOLF
K. YosHIDA

\section{SUPPORTING INSTITUTIONS}

UNIVERSITY OF ARIZONA

UNIVERSITY OF BRITISH COLUMBIA

CALIFORNIA INSTITUTE OF TECHNOLOGY

UNIVERSITY OF CALIFORNIA

MONTANA STATE UNIVERSITY

UNIVERSITY OF NEVADA, RENO

NEW MEXICO STATE UNIVERSITY

OREGON STATE UNIVERSITY
UNIVERSITY OF OREGON UNIVERSITY OF SOUTHERN CALIFORNIA STANFORD UNIVERSITY UNIVERSITY OF HAWAII UNIVERSITY OF TOKYO UNIVERSITY OF UTAH WASHINGTON STATE UNIVERSITY UNIVERSITY OF WASHINGTON 


\section{Pacific Journal of Mathematics}

Vol. 100, No. $1 \quad$ September, 1982

Charalambos D. Aliprantis, Owen Sidney Burkinshaw and M. Duhoux,

Compactness properties of abstract kernel operators $\ldots \ldots \ldots \ldots \ldots \ldots 1$

Roger C. Alperin, Locally compact groups acting on trees .............23

Robert F. Brown, Real homology of Lie group homomorphisms ......... 33

Karen Chase, Maximal groups in sandwich semigroups of binary relations . . 43

W. Wistar (William) Comfort and T. Soundararajan, Pseudocompact

group topologies and totally dense subgroups $\ldots \ldots \ldots \ldots \ldots \ldots \ldots 61$

M. Ferri and C. Gagliardi, Crystallisation moves $\ldots \ldots \ldots \ldots \ldots \ldots \ldots$

Kenneth R. Goodearl, Directly finite aleph-nought-continuous regular

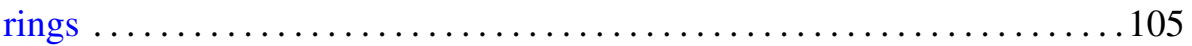

Edward Lewis Green, On the representation theory of rings in matrix

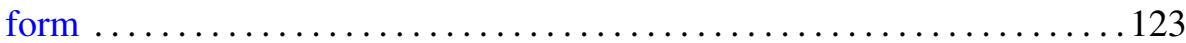

Walter Hengartner and Glenn E. Schober, Interpolation, continuation, and

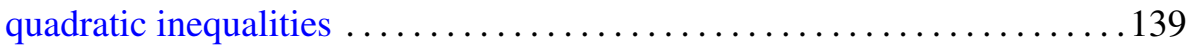

Kenneth Kunen and Haskell Paul Rosenthal, Martingale proofs of some geometrical results in Banach space theory $\ldots \ldots \ldots \ldots \ldots \ldots \ldots \ldots \ldots \ldots$

Brian William McEnnis, Shifts on indefinite inner product spaces. II . . . . . 177

Roman Pol, Note on the spaces $P(S)$ of regular probability measures whose topology is determined by countable subsets $\ldots \ldots \ldots \ldots \ldots \ldots \ldots \ldots 185$

Joan Manuel Verdera Melenchón, Finitely generated projective extensions

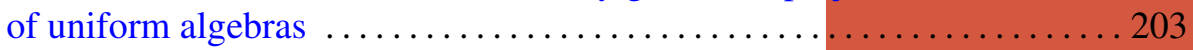

Cheng Ye You, Fixed point classes of a fiber map .................. 217 